\title{
$\mathrm{AHP}$ 를 이용한 기업별 안전평가 기법에 관한 연구
}

\section{A Study on the Method of Safety Condition Evaluation using Analytic Hierarchy Process}

\author{
백신원," • 이미선*
}

Paik, Shinwon · Lee, Misun

\begin{abstract}
In the current domestic, many industrial accidents have happened. And these are analyzed according to several factors. But it is difficult that they evaluate their business safety. Thus, we conducted a study on business specific safety assessment techniques in order that business know their safety level and perform appropriative safety activities. Study methods are survey and AHP (Analytic Hierarchy Process). Each specific weighting factors to calculate the survey was conducted for safety and consulting experts (20 persons). Weight factor was used to AHP decision support as one of the ways through a number of alternatives to the ratings for the Minesota Multiphasic. Factors are each type of industry, specific industrial scale, disaster type and strength, worker age and tenure period, and region. First, survey was conducted with 20 professionals to estimate the weighting factors. Weights between factors using the AHP analysis tool based on the mean values were calculated. Second, last 3 years between the industrial accidents statistics were used to calculate the weights for each of the rating factors in the Occupational Safety and Health Agency. Grade weights between each factor which was based on the rating of each factor was calculated as the average of three years. Finally, the weights between each factor and the grade weights for each factor using the safety level of the enterprise were calculated so that you can evaluate the weighting.
\end{abstract}

Keywords: Analytic hierarchy process; Weighting factor; Grade weight; Safety management

\section{I. 서 론}

국내에서는 해마다 많은 산업재해가 발생하고 있고 이러한 산 업재해를 여러 가지 요인별로 분석하고 통계화 하여 보고하고 있다 (Korea Occupational Safety and Health Agency, 2008 2010). 이 때문에 우리는 어떤 특정 분야 혹은 특정 규모에서 어떤 재해가 많이 일어나는지 등에 대한 내용을 쉽게 확인할 수 있지만 기업체 내에서는 자사의 안전평가를 할 수 있는 기준이 모호한 상황이다. 그렇기 때문에 기업은 자사의 안전 수준에 대 해 재해율을 이용하여 기업체간 비교 정도만 가능할 뿐 기업체 의 개별적인 특성을 반영하기 힘들고 적합한 안전 활동 계획을 수립하고 수행하는데 어려움이 있다. 이는 또한 기업이 추상적이 고 맹목적인 안전 활동을 하게 되는 이유가 되고 있다.

개별작업자에 대해서는 작업자 스스로 자율적인 안전활동이 전

\footnotetext{
* 한경대학교 토목안전환경공학과

$\dagger \quad$ Corresponding author Tel.: +82-31-670-5281

Fax: +82-31-670-5289

E-mail: paiksw@hknu.ac.kr
}

2013년 3월 7일 투고

2013년 5월 2일 심사완료

2013년 5월 6일 게재확정
개되도록 할 수 있는 안전평가모델이 개발되어 있으나 기업단위 에서는 기업특성과 그 특성의 중요도를 고려한 안전수준 평가는 이루어지고 있지 않다. 대개는 기업특성을 반영할 수 있는 주요 인자를 대상으로 안전수준을 평가하는 것은 전문가 집단의 정성 적인 점검표에 의한 것들로 수행되고 있다.

본 연구에서는 전문가 집단이 정성적인 점검표에 의해 수행하 고 있는 과정을 기업이 자체적으로 안전수준을 평가할 수 있도 록 전문가의 의견에 대해서는 설문조사를 실시하고, 그 결과를 $\mathrm{AHP}$ 기법을 구축하여 모델을 제시하는데 그 목적을 두었다.

\section{II. 이론적 고찰}

\section{1. 선행연구}

이전에 행해졌던 $\mathrm{GO}$ 의 연구에는 산업재해 사례를 대상으로, 기존의 통계적 기법이 아닌 새로운 다변량 분석기법 중 수량화 분석기법을 이용하여 재해를 분석한 결과 기존의 단순 빈도분석 이나 상관관계를 구해 얻은 결과와는 많은 차이가 있었다. 무엇 보다도 재해사고의 발생빈도와 근로손실일수와는 대부분 일치하 지 않았다는 것이며 이로서 중대재해와 사망재해의 효과적 예 
방을 위한 방향조정이 요구된다고 하였다. 이러한 점에서 수량화 분석은 새로운 관점에서 재해요인의 원인을 분석하여 중대재해 와 사망재해의 대책을 설정하는 데 매우 유용하게 활용 될 수 있 다고 제시하였다 (Byungin Go, 1999).

Song은 국제물류주선업체가 정기해운선사를 선택함에 있어 어 떠한 요인들이 중요시되는가를 $\mathrm{AHP}$ 를 이용하여 그 요인별 우선 순위를 도출하고, 정기해운선사별 요인의 중요도를 평가함으로써 그들의 고객인 국제물류주선업체의 요구를 효율적으로 충족시킬 수 있는 방안을 수립하는 기초자료를 제공하였다 (Sunok Song, 2011).

$\mathrm{Bae}$ 의 연구에서는 객관적으로 파악 가능한 농촌어메니티 자원 요소를 중심으로 일정한 기준과 형성틀에 의해 선정-분류하여 가치 평가체계를 구성하고 전문가의 의견을 반영하여 각 어메니 티 자원별 중요도를 산정한 후 $\mathrm{AHP}$ 를 이용하여 종합적인 면수 준 농촌어메니티 가치를 평가 하였고 (Seungjong Bae et al., 2007), Park의 연구에서는 교량대안들로부터 최적의 대안을 선 정하는 모델을 제시할 때와 (Jangho Park et al., 2009), Lee의 연구에서는 조선업 선종별 위험도 및 안전보건관리에 필요한 예 산수요를 비교함에 있어 AHP기법을 이용하였다 (Jongbin Leek et al., 2012).

\section{2. $\mathrm{AHP}$ (계층분석기법)}

평가 항목의 우선순위 선정은 항목간의 상대적 중요도를 감 안하여 한 명 혹은 여러 명의 의사 결정자가 참여하는 다기준 (Multiple-criteria)의 의사결정 문제를 해결하기 위해 Thomas L Saaty 교수가 고안한 모델이다. AHP는 복잡한 문제를 의사 결정자가 관리가 용이한 하위문제들로 그룹화 하는 방법으로 시 스템적 접근법에서 널리 쓰여 졌다. AHP기법은 평가자로 하여 금 쌍대 비교를 통해서 한 번에 둘 씩 비교하게 함으로써 평가 를 수월하게 한다. 또한 일관성 지수를 적용하여 평가자의 의견 에 관한 일관성을 검증함으로써 평가자의 의견이 판단에 직접 적용가능한지에 대한 사전검증 효과를 갖게 된다.

첫 번째로 문제 정의와 목표 설정이 이루어진 후 계층 구조를 만들게 된다. 그 다음 쌍대 비교를 통하여 비교행렬을 작성하게 되는데 쌍대 비교 시 중요도는 Table 1에 자세히 나타내었다. 중요도는 1 9점까지 나타낼 수 있지만 보통 1 5점 혹은 1 7점까지 나타낸다. 중요도를 부여한 뒤 Table 2처럼 비교 행렬 을 작성한다. 이 행렬을 토대로 AHP분석 도구를 이용하여 각 요인별 가중치를 계산한다.

그 다음 일관성지수 (C.I; Consistency Index)는 식 (1)), 일 관성 비율 (C,R; Consistency Rate)은 식 [2])를 통해 구한다.
Table 1 Importance for pairwise comparisons

\begin{tabular}{c|l}
\hline Importance & \multicolumn{1}{c}{ Meaning } \\
\hline \hline 1 & The same important \\
\hline 3 & Slightly more important \\
\hline 5 & Considerably more important \\
\hline 7 & Much more important \\
\hline $2,4,6,8$ & Absolutely more important \\
\hline
\end{tabular}

Table 2 The relative importance

\begin{tabular}{c|c|c|c|c|c}
\hline Factor level & Factor 1 & Factor 2 & Factor 3 & Factor 4 & Factor 5 \\
\hline \hline Factor 1 & 1 & 2 & 5 & 4 & 7 \\
\hline Factor 2 & $1 / 2$ & 1 & 3 & 5 & 6 \\
\hline Factor 3 & $1 / 5$ & $1 / 3$ & 1 & 4 & 5 \\
\hline Factor 4 & $1 / 4$ & $1 / 5$ & $1 / 4$ & 1 & 6 \\
\hline Factor 5 & $1 / 7$ & $1 / 6$ & $1 / 5$ & $1 / 6$ & 1 \\
\hline
\end{tabular}

Table 3 R.I values according to the $\mathrm{n}$ value

\begin{tabular}{c|c|c|c|c|c|c|c|c|c}
\hline $\mathrm{n}$ values & 2 & 3 & 4 & 5 & 6 & 7 & 8 & 9 & 10 \\
\hline \hline R.I values & 0 & 0.58 & 0.90 & 1.12 & 1.24 & 1.32 & 1.41 & 1.45 & 1.51 \\
\hline
\end{tabular}

$$
C . I=\frac{\left(\lambda_{\max }-n\right)}{(n-1)}
$$

$$
C \cdot R=\frac{\text { C.I }}{\text { R.I }}
$$

단, R.I 값은 $\mathrm{n}$ 값에 따라 달라지기 때문에 Table 3 에 의해 구한다.

$$
C . R \leq 0.1
$$

식 (3)이 성립하면 중요도 결정에 있어서 일관성이 있는 것으 로 판정한다. 마지막 단계는 가중치계산결과에 따라 대안을 선 택하는 것이다.

\section{III. 본 론}

\section{1. 산업형태별 가중치 분석}

본 연구에서는 기업의 안전수준 평가를 위해 산업형태별, 산 업규모별, 발생형태별, 재해정도 (치료예상기간)별, 작업자 연령 별, 재해발생시기별, 입사근속 기간별, 지역별 등 8 가지 요인을 선정하였다. 그리고 산업안전보건공단에서 각 요인별로 3 개년 간 
Table 4 Weighting value of industrial type

\begin{tabular}{c|r|c}
\hline Type & Average & Weighting value \\
\hline \hline Mining & 38.81 & 0.44789 \\
\hline Manufacturing Industry & 4.80 & 0.0554 \\
\hline Elec. Gas, municipal water & 0.87 & 0.01004 \\
\hline Construction & 3.49 & 0.04028 \\
\hline Transp. Storage, Comm. & 2.99 & 0.03451 \\
\hline Forestry & 15.82 & 0.18257 \\
\hline Fishery & 9.62 & 0.11102 \\
\hline Agriculture & 7.08 & 0.08171 \\
\hline Banking and Insurance & 0.44 & 0.00508 \\
\hline Etc. Industry & 2.73 & 0.03151 \\
\hline
\end{tabular}

Table 5 Weighting value of industrial size

\begin{tabular}{c|c|c}
\hline Type & Average & Weighting value \\
\hline \hline$<5$ & 1.66 & 0.29802 \\
\hline $5 \sim 9$ & 1.06 & 0.19031 \\
\hline $10 \sim 29$ & 0.83 & 0.14901 \\
\hline $30 \sim 49$ & 0.60 & 0.10772 \\
\hline $50 \sim 99$ & 0.47 & 0.08438 \\
\hline $100 \sim 299$ & 0.32 & 0.05745 \\
\hline $300 \sim 499$ & 0.22 & 0.0395 \\
\hline $500 \sim 999$ & 0.17 & 0.03052 \\
\hline$>1,000$ & 0.24 & 0.04309 \\
\hline
\end{tabular}

Table 6 Weighting value of typical occurrence

\begin{tabular}{c|r|r}
\hline Type & Average & Weighting value \\
\hline \hline Fall & 13,885 & 0.14251 \\
\hline Overturning & 19,984 & 0.20511 \\
\hline Collision & 8,133 & 0.08347 \\
\hline Falling and Fly & 8,302 & 0.08521 \\
\hline Collapse and Destruction & 922 & 0.00946 \\
\hline Cramped & 16,102 & 0.16526 \\
\hline Cur and picking & 7,301 & 0.07493 \\
\hline Electric shock & 457 & 0.00469 \\
\hline Explosion & 432 & 0.00443 \\
\hline Bursting & 96 & 0.001 \\
\hline Fire & 447 & 0.00459 \\
\hline Abnormal temp & 2,358 & 0.0242 \\
\hline Drwoned & 34 & 0.00035 \\
\hline Toxication and choke & 425 & 0.00436 \\
\hline Mining collapse & 1 & 0.00001 \\
\hline Beyond movement & 2,101 & 0.02156 \\
\hline Traffic accident & 4,970 & 0.051 \\
\hline Sick due to occupational case & 8,753 & 0.08984 \\
\hline etc. & 2,397 & 0.0246 \\
\hline Not classfied & 333 & 0.00341 \\
\hline & &
\end{tabular}

Table 7 Weighting value of duration of treatment

\begin{tabular}{c|c|c}
\hline Type & Average & Weighting value \\
\hline \hline Death & 2,268 & 0.02328 \\
\hline over 6 month & 14,967 & 0.15363 \\
\hline 91 $\sim$ 180 days & 30,460 & 0.31265 \\
\hline $29 \sim 90$ days & 37,083 & 0.38063 \\
\hline $15 \sim 28$ days & 7,865 & 0.08073 \\
\hline $8 \sim 14$ days & 2,762 & 0.02835 \\
\hline 4 $\sim$ days & 2,020 & 0.02073 \\
\hline
\end{tabular}

Table 8 Weighting value of age of employee

\begin{tabular}{c|r|c}
\hline Age & Average & Weighting value \\
\hline \hline under 18 & 173 & 0.00178 \\
\hline $18 \sim 24$ & 3,644 & 0.0374 \\
\hline $25 \sim 29$ & 7,457 & 0.07654 \\
\hline $30 \sim 34$ & 8,951 & 0.09187 \\
\hline $35 \sim 39$ & 10,687 & 0.10969 \\
\hline $40 \sim 44$ & 12,754 & 0.13091 \\
\hline $45 \sim 49$ & 14548 & 0.14932 \\
\hline $50 \sim 54$ & 14,978 & 0.15374 \\
\hline $55 \sim 59$ & 11,015 & 0.11306 \\
\hline at least 60 & 13,218 & 0.13561 \\
\hline Not classified & 2 & 0.00002 \\
\hline
\end{tabular}

Table 9 Weighting value of period of disaster

\begin{tabular}{c|c|c}
\hline Month & Average & Weighting value \\
\hline \hline Jan & 7,706 & 0.0791 \\
\hline Feb & 6,791 & 0.06971 \\
\hline Mar & 7,929 & 0.08139 \\
\hline Apr & 7,975 & 0.08186 \\
\hline May & 7,644 & 0.07846 \\
\hline Jun & 8,495 & 0.0872 \\
\hline Jul & 9,099 & 0.0934 \\
\hline Aug & 7,800 & 0.08006 \\
\hline Sep & 8,114 & 0.08329 \\
\hline Oct & 8,331 & 0.08551 \\
\hline Nov & 8,627 & 0.08855 \\
\hline Dec & 8,913 & 0.09149 \\
\hline
\end{tabular}

의 통계자료의 평균값을 계산하였다 (Korea Occupational Safety and Health Agency, 2008-2010)

산업형태별 가중치를 분석한 결과는 Table 4 와 같다.

\section{2. 기업별 안전수준 평가를 위한 $\mathrm{AHP}$ 모델}

산업재해 분석 자료로 부터 8가지 요인을 선정하였다 (Korea 
Table 10 Weighting value of duration of employment

\begin{tabular}{c|c|c}
\hline Period & Average & Weighting value \\
\hline \hline less 6 months & 51,754 & 0.53123 \\
\hline 6 month $\sim$ year & 10,095 & 0.10362 \\
\hline 1 2 year & 9,713 & 0.0997 \\
\hline 2 3 year & 5,508 & 0.05654 \\
\hline 3 4 year & 3393 & 0.03483 \\
\hline 4 $\sim 5$ year & 2,473 & 0.02538 \\
\hline 5 10 year & 6,827 & 0.07008 \\
\hline at least 10 year & 7,228 & 0.07419 \\
\hline Not classified & 432 & 0.00443 \\
\hline
\end{tabular}

Table 11 Weighting value of location of company

\begin{tabular}{c|c|c}
\hline Area & Average & Weighting value \\
\hline \hline Seoul & 0.41 & 0.03581 \\
\hline Busan & 0.83 & 0.07249 \\
\hline Daegu & 0.82 & 0.07162 \\
\hline Incheon & 0.82 & 0.07162 \\
\hline Gwangju & 0.82 & 0.07162 \\
\hline Daejeon & 0.68 & 0.05939 \\
\hline Gangwon & 0.98 & 0.08559 \\
\hline Gyeonggi & 0.81 & 0.07074 \\
\hline Chungbuk & 0.81 & 0.07074 \\
\hline Chungnam & 0.66 & 0.05764 \\
\hline Jeonbuk & 0.92 & 0.08035 \\
\hline Jeonnam & 0.72 & 0.06289 \\
\hline Kyeongbuk & 0.61 & 0.05328 \\
\hline Kyeongnam & 0.85 & 0.07424 \\
\hline Cheju & 0.71 & 0.06201 \\
\hline
\end{tabular}

Occupational Safety and Health Agency, 2008-2010). 주요 요인으로 산업형태별, 산업규모별, 발생형태별, 재해정도 (치료예 상기간)별, 작업자연령별, 재해발생시기별, 작업자 입사근속 기간 별, 지역별이다.

8 가지 요인별 가중치 분석을 위해 전문가에게 설문조사를 실 시하였다. 전문가 집단은 안전관리 관련 경력이 5년 이상 있는 자, 안전관리 박사학위 소지자로 교육경험이 있는 자 20명으로 구성하였다. 설문조사와 AHP기법을 통해 기업의 안전 수준을 평가하는데 중요하다고 생각되는 8 가지 요인의 가중치를 산정 결과, 산업형태 0.239 , 산업규모 0.156 , 발생형태 0.323 , 재해정 도 0.112 , 작업자연령 0.046 , 재해발생시기 0.069 , 입사근속기간 0.032 , 지역 0.024 로 계산되었다.

$\mathrm{AHP}$ 프로그램을 이용하여 가중치 산정을 한 결과 C.I (Consistency index) 값은 0.0536으로 계산되었다. 이 값을 토
대로 C.R값을 계산하여 8가지 요인별 중요도 결정에 일관성이 있는지 확인해 보았는데 0.038 로 계산되어 0.1 보다 작기 때문에 8 가지 요인의 가중치 산정에 있어서 일관성이 있다고 판단된다. 8 가지의 요인별 세부 요인에 대한 가중치는 계급구간 또는 시 기 등에 관련된 항목이기 때문에 각 세부요인이 주요 요인에 미 치는 영향력을 평가하기 위해서 3 개년 간의 산업재해 통계자료 (Korea Occupational Safety and Health Agency, 2008 2010)로부터 계산하고 작성된 가중치를 이용하여 기업별 안전 수준을 점수화할 수 있는 안전수준 평가모델을 개발하였다.

한편 기업별 안전 실태 평가점수는 아래 식으로 구할 수 있다.

기업별 안전 실태 평가 점수

$$
=\sum_{i=1}^{n}\left[w_{i} \sum_{j=1}^{l} x_{i j}\right] \times 100
$$

여기서, $w_{i}=1$ 차 요인의 가중치 점수

$$
x_{i j}=i \text { 번째 요인의 } 2 \text { 차 요소의 가중치 점수 }
$$

\section{AHP모델의 적용 및 고찰}

본 연구에서 제안된 모델의 적용성을 살펴보기 위하여 121 명 근로자 규모의 제조업체인 A기업의 2011년도 산업재해자료 Table 12 와 4,038명 근로자 규모의 건설업체인 B기업의 2011년도 산 업재해자료 Table 13을 토대로 두 기업의 안전수준을 점수화 하 였다. A기업과 B기업은 재해율 측면에서 볼 때는 $\mathrm{A}$ 기업의 재해 율이 높게 나오는 경우이지만, 본 모델을 적용하여 안전수준을 계산한 결과 각각 22.871, 126.801이 나왔다. B기업이 A기업에 비해 규모가 훨씬 큰 만큼 더 많은 재해가 발생하였고, 안전수준 의 점수도 $\mathrm{B}$ 기업이 5 배 이상 크게 산정되었다.

기업체의 안전수준평가방법은 재해율에 의한 기업체의 안전의 식의 관리가 갖는 한계점을 극복할 수 있을 것으로 보이며, 중대 재해를 유발할 수 있는 큰 점수의 안전수준 점수를 보이는 기업 체일수록 더욱 더 철저한 관리 감독의 필요성을 제기하고 있다 고 볼 수 있다.

Table 12 Accidents status of A company (2011)

\begin{tabular}{c|c|c|c|c|c}
\hline Onset & Occurring form & $\begin{array}{c}\text { Disaster } \\
\text { degree }\end{array}$ & Age & Longevity & Area \\
\hline \hline Jan. 17 & Fall & 4 weeks & 44 & 1 year & Chungbuk \\
\hline Feb. 19 & Car accident & 3 weeks & 48 & 6 month $\sim 1$ year & Chungbuk \\
\hline Mar. 8 & Cut off & 2 months & 30 & 3 months & Chungbuk \\
\hline
\end{tabular}


Table 13 Accidents status of B company (2011)

\begin{tabular}{c|c|c|c|c|c}
\hline Onset & Occurring form & $\begin{array}{c}\text { Disaster } \\
\text { degree }\end{array}$ & Age & Longevity & Area \\
\hline \hline Jan. 4 & flying & 2 weeks & 44 & 2 years & Kangwon \\
\hline Jan. 8 & Collision & 2 weeks & 35 & $1 \sim 2$ years & Jeongbuk \\
\hline Jan. 18 & Fall & 4 weeks & 50 & less than 6 months & Gyeonggi \\
\hline Mar. 22 & Fall & 3 months & 43 & $2 \sim 3$ years & Kyungbuk \\
\hline Apr. 15 & Narrowness & 3 months & 36 & 6 months $\sim 1$ year & Gyeonggi \\
\hline Apr. 19 & Fall & 4 months & 47 & 6 months $\sim 1$ year & Kyungbuk \\
\hline Jun. 20 & Car accident & 2 weeks & 29 & 1 year & Gyeonggi \\
\hline Jun. 21 & Collision & 3 weeks & 55 & 2 year & Kangwon \\
\hline Jul. 23 & Car accident & 4 weeks & 50 & 6 months $\sim 1$ year & Chungnam \\
\hline Aug. 12 & Fall & 2 months & 37 & $1 \sim 2$ years & Kyungbuk \\
\hline Aug. 19 & Narrowness & 2 months & 46 & less than 6 months & Chungnam \\
\hline Sept. 7 & Fall & Death & 53 & 6 months $~ 1$ year & Chungnam \\
\hline Sept. 7 & Fall & 4 months & 58 & 1 year & Chungnam \\
\hline Sept. 7 & Fall & 2 months & 48 & less than 6 months & Chungnam \\
\hline Sept. 7 & Flying & 4 weeks & 40 & 6 months $~ 1$ year & Chungnam \\
\hline Sept. 30 & Car accident & 2 weeks & 49 & 3 year & Chungnam \\
\hline Oct. 24 & Electric shock & 4 weeks & 53 & less than 6 months & Kyungnam \\
\hline Nov. 8 & Car accident & 3 weeks & 49 & $1 \sim 2$ years & Jeju \\
\hline
\end{tabular}

\section{IV. 결 론}

본 연구는 기업이 자사의 안전수준을 정확히 파악하여 기업에 맞는 안전 활동을 수립할 수 있도록 하는 데에 목표를 두고 진행 하였다.

설문조사와 AHP기법을 통해 기업의 안전 수준을 평가하는데 중요하다고 생각되는 8 가지 요인의 가중치를 산정하였다. 그 결 과, 산업형태 0.239 , 산업규모 0.156 , 발생형태 0.323 , 재해정 도 0.112 , 작업자연령 0.046 , 재해발생시기 0.069 , 입사근속기 간 0.032 , 지역 0.024 로 계산되었다. 또한 8 가지 요인의 등급 간 가중치도 산정하였다.

이 결과를 토대로 실제 기업의 안전수준을 평가해보았다. $\mathrm{A}$ 기 업의 안전수준을 평가한 결과 22.871로 계산되었고 B기업의 안 전수준을 평가한 결과 126.801 로 계산되었다. 두 기업의 안전수 준을 비교했을 때 $\mathrm{B}$ 기업의 안전수준의 점수가 훨씬 높게 나타났 지만 $\mathrm{A}$ 기업이 $\mathrm{B}$ 기업보다 더 안전하다고 단정해서 말하는 것은
문제가 있을 수 있다. 그 이유는 두 기업의 업종이 다를 뿐 아니 라 기업의 규모에도 상당한 차이가 있기 때문이다. 따라서 안전 수준 점수만 가지고 기업의 안전수준을 평가하기에는 무리가 있 을 수 있으나 동일업종의 비슷한 규모의 기업간의 안전수준 비 교에는 적용가능하고 활용할 수 있을 것으로 사료된다. 따라서 기업으로 하여금 자사의 안전에 대한 기준을 세밀하게 수립하고 안전수준을 정확히 알게 하여 기업이 적합한 안전 활동을 계획 하고 수행하는 데에 도움을 줄 수 있을 것으로 판단된다.

향후 연구에서는 산업별, 기업규모별 안전수준 평가 등급표를 작성하여 기업이 자사의 안전수준을 평가할 수 있도록 하는 연 구가 진행되어야 할 것이다.

\section{REFERENCES}

1. Byungin, G., 1999. Disaster Statistical Analysis Using Multivariate Analysis Techniques. Spring Conference of the Korean Society of Safety 40 (in Korean).

2. Sunok Song, 2011. A Study on the Selection of the International Ship Logistics Usging the AHP. Journal of Korea Association for International Commerce and Information 13(2): 95-117 (in Korean).

3. Bae, S., and H. Chung, 2007. Development of a Rural Amenity Values Assessment Model by Analytic Hierarchy Process. Journal of the Korean Society of Agricultural Engineers 49(5): 33-44 (in Korean).

4. Park, J., Y. Shin, Y. Ahn, and K. Lee 2009. Measurement of Value Engineering for Bridge Superstructures Using Anlytic Hierarchy Process. Journal of the Korean Society of Safety 24(6): 105-110 (in Korean).

5. Lee, J., and S. Chang, 2012. Measurement of Severity of Hazards and Investment in Occupational Safety \& Health According to Ship Types Using Analytic Hierarchy Process. Journal of the Korean Society of Safety 27(1): 105-110 (in Korean).

6. Korea Occupational Safety and Health Agency, 20082010, Analysis of Industrial Accidents (in Korean). 